2)In our study, ILD was more frequently associated with women and joint or skin symptoms, as well as with data on immunological activity, without statistical significance.

REFERENCES:

[1] Aguilera-Pickens G, Abud-Mendoza C. Pulmonary Manifestations in Systemic Lupus Erythematosus: Pleural Involvement, Acute Pneumonitis, Chronic Interstitial Lung Disease and Diffuse Alveolar Hemorrhage. Clinical rheumatology. Vol.14 Issue. 5. Pages 294-300 (September-October 2018).

Disclosure of Interests: None declared

DOI: 10.1136/annrheumdis-2021-eular.2726

\section{POS0753 SUBSPECIALTY LUPUS CLINIC CARE IS ASSOCIATED WITH HIGHER QUALITY FOR PATIENTS WITH SYSTEMIC LUPUS ERYTHEMATOSUS}

S. Sreedharan ${ }^{1,2}$, A. Hoi ${ }^{1,2}$, N. Li ${ }^{2}$, G. Littlejohn ${ }^{2}$, R. Buchanan ${ }^{3}$, M. Nikpour ${ }^{4}$,

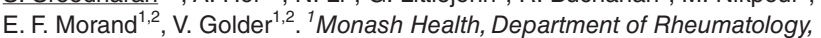
Melbourne, Australia; ${ }^{2}$ Monash University, School of Clinical Sciences, Melbourne, Australia; ${ }^{3}$ Austin Hospital, Department of Rheumatology, Heidelberg, Australia; ${ }^{4}$ St. Vincent's Hospital Melbourne, Department of Rheumatology, Fitzroy, Australia

Background: Healthcare quality for systemic lupus erythematosus (SLE) is a modifiable target for improving patient outcomes. Disease-specific subspecialty clinics offer experienced healthcare professionals, collaborative multidisciplinary teams and streamlined care processes. A single centre study in the USA has suggested superior performance of the subspecialty lupus clinic in the provision of quality care (1), but this has not been examined outside the USA where access to care may be influential.

Objectives: To assess the quality of SLE care provided in a subspecialty lupus clinic compared with hospital general rheumatology and private rheumatology clinics in a non-US, universal healthcare setting.

Methods: Lupus patients $(n=258)$ were recruited in 2016 from various clinic settings in Australia, including a subspecialty lupus clinic $(n=147)$, two hospital general rheumatology clinics $(n=56)$ and two private clinics $(n=55)$. Quality of care was assessed using 31 validated SLE quality indicators (QI) encompassing diagnostic work-up, disease and comorbidities assessment, drug monitoring, preventative care and reproductive health $(2,3)$. Data were collected from medical records and patient questionnaires. Overall and individual QI performance was calculated and compared between the three clinic settings, and multivariable regression was performed to adjust for sociodemographic, disease and healthcare factors.

Results: Median [IQR] overall performance on eligible QIs was higher in the lupus clinic $(66.7 \%$ [16.9]) than the hospital general rheumatology (52.7\% [10.6]) and private rheumatology $(50.00 \%$ [18.0]) clinics $(p<0.01)$, and remained significant with multivariable adjustment. This trend was still observed when the overall performance was reassessed to include patient self-report $(73.1 \%$ [14.8] vs $68.1 \%$ [11.5] vs $63.2 \%$ [13.4], $p<0.01)$. This difference may be due to consistent formal assessments of disease activity ( $100 \%$ vs $0 \%$ vs $0 \%, p<0.01)$ and disease damage $(95.9 \%$ vs $0 \%$ vs $0 \%, p<0.01$ ) at the lupus clinic. Performance was high across all clinic settings for diagnostic work-up, comorbidity assessment, drug monitoring, prednisolone taper, osteoporosis management, lupus nephritis and pregnancy quality indicators. However, the lupus clinic significantly outperformed the other clinic settings on eligible quality indicators for new medication counselling, pre-immunosuppression hepatitis and tuberculosis screening, drug toxicity assessment, sun avoidance education, vaccinations, cardiovascular risk factor assessment and contraception counselling. Conclusion: SLE patients managed in a subspecialty lupus clinic received higher overall quality of care when compared to hospital general rheumatology and private rheumatology clinics. Regular assessment of QI performance can improve quality of care for patients in all clinic settings.

REFERENCES:

[1] Arora, S et al. Does Systemic Lupus Erythematosus Care Provided in a Lupus Clinic Result in Higher Quality of Care Than That Provided in a General Rheumatology Clinic? Arthritis Care Res. 2018;70(12):1771-1777.

[2] Mosca, M et al. Development of quality indicators to evaluate the monitoring of SLE patients in routine clinical practice. Autoimmune Rev. 2011;10(7):383-8.

[3] Yazdany, J et al. A quality indicator set for systemic lupus erythematosus. Arthritis Rheum. 2009;61(3):370-7.

Disclosure of Interests: None declared

DOI: 10.1136/annrheumdis-2021-eular.2760

\section{POS0754 \\ DEVELOPMENT OF A RISK PREDICTION MODEL FOR VENOUS THROMBOEMBOLISM IN PATIENTS WITH SYSTEMIC LUPUS ERYTHEMATOSUS: THE SLE-VTE SCORE}

H. You ${ }^{1,2}$, M. Li ${ }^{1}$, J. L. Zhao ${ }^{1}$, L. Wu ${ }^{3}$, X. Duan ${ }^{4}$, H. Luo ${ }^{5}$, C. Zhao ${ }^{6}$, F. Zhan ${ }^{7}$,

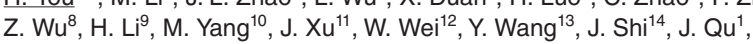

Q. Wang ${ }^{1}$, X. Leng ${ }^{1}$, X. Tian ${ }^{1}$, Y. Zhao ${ }^{1}$, X. Zeng ${ }^{1} .{ }^{1}$ Peking Union Medical College Hospital, Peking Union Medical College \& Chinese Academy of Medical Sciences, Department of Rheumatology, Beijing, China; ${ }^{2}$ The First Affiliated Hospital of Nanjing Medical University, Department of Rheumatology, Nanjing, China; ${ }^{3}$ People Hospital of Xinjiang Uygur Autonomous Region, Department of Rheumatology, Urumchi, China; ${ }^{4}$ The Second Affiliated Hospital of Nanchang University, Department of Rheumatology, Nanchang, China; ${ }^{5}$ Xiangya Hospital, Central South University, Department of Rheumatology, Changsha, China; ${ }^{6}$ The First Affiliated Hospital of Guangxi Medical University, Department of Rheumatology, Nanning, China; ${ }^{7}$ Hainan General Hospital, Department of Rheumatology, Haikou, China; ${ }^{8}$ Xijing Hospital Affiliated to the Fourth Military Medical University, Department of Rheumatology, Xi'an, China: ${ }^{9}$ Affiliated Hospital of Inner Mongolia Medical College, Department of Rheumatology, Hohhot, China; ${ }^{10}$ Nanfang Hospital, Southern Medical University, Department of Rheumatology, Guangzhou, China; ${ }^{11}$ First Affiliated Hospital of Kunming Medical University, Department of Rheumatology, Kunming, China; ${ }^{12}$ Tianjin Medical University General Hospital, Department of Rheumatology, Tianjin, China; ${ }^{13}$ Institute of Basic Medical Sciences, China Academy of Medical Sciences \& Peking Union Medical College, Department of Epidemiology and Bio-Statistics, Beijing, China; ${ }^{14}$ Peking Union Medical College Hospital, Peking Union Medical College \& Chinese Academy of Medical Sciences, Department of Respiratory and Critical Care Medicine, Beijing, China

Background: Patients with systemic lupus erythematosus (SLE) have a substantially increased risk of venous thromboembolism (VTE). An individual VTE risk assessment is important to ensure that all patients are assessed and given adequate thromboprophylaxis.

Objectives: We conducted this study to develop a risk score for VTE in patients with SLE.

Methods: Patients with SLE who participated in the Chinese SLE Treatment and Research group were enrolled in this study. Patient baseline information and clinical laboratory indicators were obtained, and VTE events were recorded every 3-6 months during follow-up visits. The risk prediction model was created and internally validated using the bootstrap methods, and a scoring system was established (Figure 1).

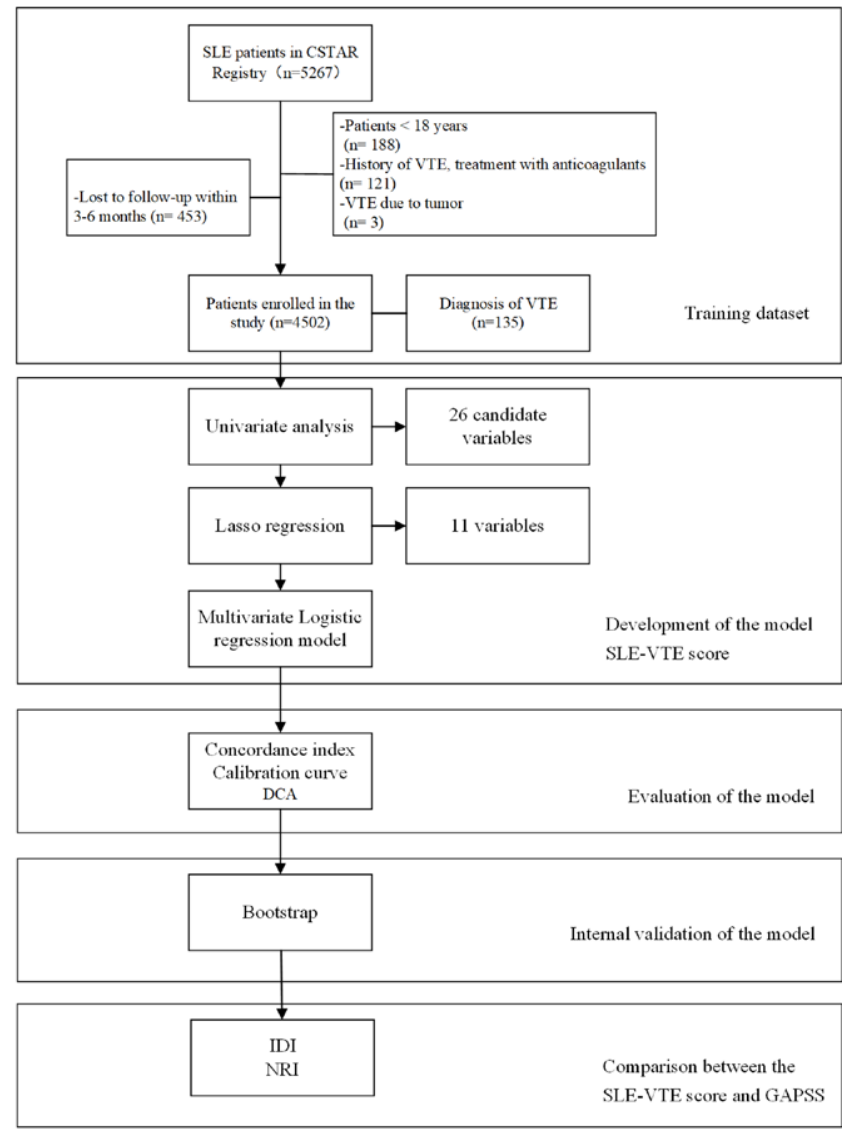

Figure 1. Flow chart of study design. 
Results: Out of 4,502 patients included in this study, 135 had a VTE event. After univariate analysis and Lasso regression, the following 11 variables were identified and included in the risk prediction model: male sex, age, BMI $\geq 25 \mathrm{~kg} / \mathrm{m} 2$, hyperlipidemia, hypoalbuminemia, hsCRP $>3 \mathrm{mg} / \mathrm{L}$, renal involvement, nervous system involvement, anti- $\beta 2$-glycoprotein I antibody positivity, lupus anticoagulant positivity, and no use of hydroxychloroquine. The AUC for the SLE-VTE score (Table 1) was 0.947 ( $95 \% \mathrm{Cl}, 0.9249-0.9694)$. The SLE-VTE score's sensitivity and specificity with the optimal cutoff value of 13 were 0.919 and 0.881 , respectively. The SLE-VTE score was superior to the GAPSS system in predicting the risk of VTE in patients with SLE $(A \cup C=0.947$ vs. $0.680, P<0.001$; integrated discrimination improvement $(I D I)=0.6652, P<0.001$; net reclassification improvement $(\mathrm{NRI})=0.6652$, $\mathrm{P}<0.001)$.

Table 1. Final multivariable analysis for venous thromboembolism risk in patients with SLE

\begin{tabular}{|c|c|c|c|c|}
\hline & $\begin{array}{c}\beta \\
\text { coefficients }\end{array}$ & $\begin{array}{l}\text { Odds ratio* } \\
(95 \% \mathrm{Cl})\end{array}$ & $P$-value & $\begin{array}{c}\text { Points in } \\
\text { scoring system }\end{array}$ \\
\hline Male & 0.621 & $\begin{array}{c}1.86 \\
(0.953-3.503)\end{array}$ & 0.061 & 2 \\
\hline Age at study entry $(\geq 50)$ & 0.837 & $\begin{array}{c}2.308 \\
(1.339-3.915)\end{array}$ & 0.002 & 3 \\
\hline BMI02( kg/m² & 0.792 & $\begin{array}{c}2.209 \\
(1.333-3.627)\end{array}$ & 0.002 & 3 \\
\hline Hyperlipemia & 0.838 & $\begin{array}{c}2.313 \\
(1.246-4.166)\end{array}$ & 0.006 & 3 \\
\hline Hypoalbuminemia & 2.163 & $\begin{array}{c}8.697 \\
(5.185-14.794)\end{array}$ & $<0.001$ & 7 \\
\hline $\mathrm{hsCRP}>3 \mathrm{mg} / \mathrm{L}$ & 1.452 & $\begin{array}{c}4.272 \\
(2.618-6.968)\end{array}$ & $<0.001$ & 5 \\
\hline Anti $\beta 2 \mathrm{GPI}$ & 1.013 & $\begin{array}{c}2.754 \\
(1.543-4.853)\end{array}$ & 0.001 & 3 \\
\hline LA & 1.559 & $\begin{array}{c}4.752 \\
(2.799-8.072)\end{array}$ & $<0.001$ & 5 \\
\hline Nervous system & 2.382 & $\begin{array}{c}10.832 \\
(6.163-18.998)\end{array}$ & $<0.001$ & 8 \\
\hline Lupus nephritis & 0.835 & $\begin{array}{c}2.305 \\
(1.414-3.756)\end{array}$ & 0.001 & 3 \\
\hline $\begin{array}{l}\text { No use of } \\
\text { hydroxychloroquine }\end{array}$ & 1.771 & $\begin{array}{c}5.876 \\
(3.722-9.401)\end{array}$ & $<0.001$ & 6 \\
\hline
\end{tabular}

BMI: body mass index; hsCRP: Hypersensitive c-reactive protein; ACL: anticardiolipin antiß2GPI: anti- $\beta 2$-glycoprotein I, LA: lupus anticoagulantm;Values in bold are statistically significant at $p<0.05$

Conclusion: Various factors are related to the occurrence of VTE in patients with SLE. The proposed SLE-VTE risk score can accurately predict the risk of VTE and help identify SLE patients with a high risk of VTE who may benefit from thromboprophylaxis.

REFERENCES:

[1] Ramirez GA, Efthymiou M, Isenberg DA, Cohen H. Under crossfire: thromboembolic risk in systemic lupus erythematosus. Rheumatology. 2018;58:940-952.

[2] Chung WS, Lin CL, Chang SN, Lu CC, Kao CH. Systemic lupus erythematosus increases the risks of deep vein thrombosis and pulmonary embolism: a nationwide cohort study. J Thromb Haemost. 2014;12:452458.

[3] Liew NC, Alemany GV, Angchaisuksiri P, et al. Asian venous thromboembolism guidelines: updated recommendations for the prevention of venous thromboembolism. Int Angiol. 2017;36:1.

[4] Savino S, Giovanni S, Veronica M, Dario R, Khamashta MA, Laura BM. GAPSS: the Global Anti-Phospholipid Syndrome Score. Rheumatology. 2013:8.

[5] Konstantinides SV, Meyer G, Becattini C, et al. 2019 ESC Guidelines for the diagnosis and management of acute pulmonary embolism developed in collaboration with the European Respiratory Society (ERS). Eur Heart J. 2020;41:543-603.

[6] Moghadamyeghaneh Z, Hanna MH, Carmichael JC, Nguyen NT, Stamos MJ. A Nationwide Analysis of Postoperative Deep Vein Thrombosis and Pulmonary Embolism in Colon and Rectal Surgery. J Gastrointest Surg. 2014;18:2169-2177.

Disclosure of Interests: None declared

DOI: 10.1136/annrheumdis-2021-eular.2769

\section{\begin{tabular}{|l|l}
\hline POS0755 PREVALENCE AND RELEVANCE OF ANTIBODIES \\
\hline
\end{tabular} AGAINST CITRULLINATED ALPHA ENOLASE (ANTI-CEP1) IN CONNECTIVE TISSUE DISEASES}

A. Alunno ${ }^{1}$, O. Bistoni ${ }^{1}$, F. Carubbi ${ }^{2}$, M. Antonucci ${ }^{1}$, S. Calvacchi ${ }^{1}$, E. Bartoloni

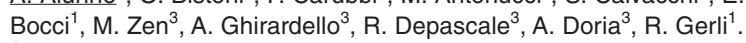

${ }^{1}$ Rheumatology Unit, University of Perugia, Department of Medicine, Perugia, Italy; ${ }^{2}$ Internal Medicine and Nephrology Unit, University of L'Aquila, Department of Life, Health and Environmental Sciences, L'Aquila, Italy;

${ }^{3}$ Division of Rheumatology, University of Padua, Department of Medicine DIMED, Padua, Italy

Background: Anti-citrullinated alpha enolase antibodies have been investigated in rheumatoid arthritis and associated with bone erosion and interstitial lung disease but little is known about their prevalence and role in connective tissue diseases (CTDs).

Objectives: The aim of this study was to investigate the prevalence and relevance of anti-CEP1 antibodies in CTDs.

Methods: Serum samples from five independent patient cohorts were assessed: 1) established (est) primary Sjogren's syndrome (pSS) $N=78,2$ ) est-systemic lupus erythematosus (SLE) $\mathrm{N}=52$, 3) est-systemic sclerosis (SSc) $\mathrm{N}=71,4$ ) pSS at disease onset $\mathrm{N}=30,5$ ) SLE at disease onset $\mathrm{N}=46$ (cohorts 4 and 5 had at least 3 years of follow-up). Samples from ninety sex and age matched healthy donors (HD) and 200 patients with est-RA (disease controls) were also tested. Anti-CEP1 IgG antibodies were measured with a commercially available ELISA kit (Euroimmun, Luebeck, Germany).

Results: Anti-CEP1 titer was significantly higher in est-pSS, est-SLE and estSSc compared to HD, significantly lower in est-pSS and est-SSc compared to est-RA and comparable in est-SLE versus est-RA. We divided patients in every CTD group based on whether their anti-CEP1 titer was below or above the 25th, 50th and 75th percentile. In est-SLE anti-CEP1 values over the 25th percentile were associated with articular involvement (odds ratio, OR $(95 \%$ confidence interval, $\mathrm{Cl})=11.5 ; 1.9-70.6, \mathrm{p}=0.008$ ). In est-pSS, no relationship between antiCEP1 $>25^{\text {th }}$ percentile and articular involvement was found but rather an association with rheumatoid factor positivity $(\mathrm{OR}(95 \% \mathrm{Cl})=4.8,1.6-14.1, \mathrm{p}=0.004)$ and salivary gland swelling $(\mathrm{OR}(95 \% \mathrm{Cl})=6.2,1.3-29.1, \mathrm{p}=0.021)$. In est-SSc no difference could be detected across the 3 groups. Anti-CEP-1 titers in pSS and SLE at onset did not differ from each other, were comparable also to those of HD and significantly lower than those of est-pSS, est-SLE and est-RA patients (all $\mathrm{p}<0.0001)$.). Of interest, we could retrieve a serum sample collected at the time of diagnosis for 5 patients from the cohort of established pSS and we observed that anti-CEP1 titers were significantly lower at pSS onset than during follow up (at least 12 months after the diagnosis, $p=0.0024$ ). No difference was observed in the clinical presentation at disease onset according to different anti-CEP1 titer and they did not predict the development of new clinical manifestations during follow-up.

Conclusion: Anti-CEP-1 antibodies can be detected in CTDs at different title during the disease course and may increase overtime, at least in pSS. Although anti-CEP1 antibodies are associated with specific clinical manifestation in est-CTDs, such as articular involvement in est-SLE, they seem to lack a predictive value for future manifestations when measured at disease onset.

REFERENCES:

[1] Alunno A, Bistoni O, Pratesi $F$ et al Rheumatology (Oxford) 2018.

[2] Manca ML, Alunno A, D'Amato C et al. Joint Bone Spine 2018.

Disclosure of Interests: None declared

DOI: 10.1136/annrheumdis-2021-eular.2825

\section{POS0756 \\ HOW HAS OUR MANAGEMENT OF SLE CHANGED OVER THE LAST THREE DECADES? REAL LIFE DATA FROM A MONOCENTRIC COHORT}

V. Signorini ${ }^{1}$, C. Tani ${ }^{2}$, E. Elefante ${ }^{2}$, D. Zucchi ${ }^{2}$, C. Stagnaro ${ }^{2}$, F. Ferro ${ }^{2}$, L. Carli ${ }^{2}$, M. Mosca ${ }^{2}{ }^{1}$ University of Pisa, Rheumatology Unit, Pisa, Italy; ${ }^{2}$ University of Pisa, Rheumatology Unit, Pisa, Italy

Background: Over the last decades new therapies have been approved and new treatment strategies have been proposed in the treatment of SLE. In particular, recently, EULAR recommendations ${ }^{1}$ and the Treat to Target $^{2}$ approach established a new paradigm for the treatment of SLE based on the early introduction of immunosuppressive treatment and glucocorticoids (GC) minimization 